\title{
Mitteilungen / Communications
}

\section{Hommage au Dr Roland Fawer}

Le décès subit du $D^{r}$ Roland Fawer prive la médecine du travail et le corps médical d'un de ses représentants les plus doués et les plus qualifiés. Agé de 37 ans, le $D^{r}$ Fawer avait obtenu son diplóme de médecin en 1973. Alliant un sens aigu de la rigueur scientifique avec un profond désir de se pencher sur les problèmes de sécurité de l'homme au trayail, il avait acquis une excellente formation de base, par des stages de médecine, de chirurgie, de pharmacologie et de médecine du travail. Au cours de ces stages, il avait effectué des travaux de premier ordre, dont certains publiés dans des revues internationales de haut niveau. Délaissant une carrière scientifique qui s'annonçait brillante et dans l'espoir d'obtenir un poste de médecin inspecteur du travail pour le canton de Vaud, il avait reçu, pour compléter sa formation en médecine du trayail, une bourse de deux ans pour l'Angleterre, financée en partie par le Fonds national suisse de la recherche scientifique et en partie par le service de santé du canton de Vaud. A l'échéance de cette période, le $D^{\prime}$ Fawer était averti que le poste envisagé ne pouvait être créé. Le $D^{r}$ Fawer a alors la chance de trouver devant lui le poste vacant de médecin-chef du service de médecine du travail à Neuchâtel. En deux ans et demi, il effectue dans cette charge un travail remarquable, ne ménageant ni son temps ni ses conseils. Il continue a publier d'excellents travaux, souvent en collaboration avec diverses équipes de médecine du travail.

Très absolu dans ses convictions, haïssant les compromis, le $D^{r}$ Fawer pouvait paraitre parfois agressif. En fait, si on le connaissait bien, il était d'une gentillesse, d'une cordialité et d'une patience peu communes.

L'exercice de la médecine du travail exige indépendance, courage, persévếrance, intégrité. Le $D^{r}$ Fawer possédait ces qualités. Encore tout récemment, n'avait-il pas préparé une mise en garde contre le projet d'introduction du travail de nuit chez les femmes de l'entreprise Ebauches SA? Rien pourtant ne l'obligeait à une telle démarche. Toute la medecine du travail regrette profondément la disparition du $\mathrm{D}^{r}$ Fawer qui, certainement, aurait continué à se mettre au service d'une cause qu'il a toujours défendue avec ardeur.

Professeur M. Lob, Lausanne

\section{Enseigner la santé}

Les résultats et recommandations tirés de l'expérience de prévention et d'éducation pour la santé réalisée à Aarau de 1978 à 1980, dans le cadre du Programme national de recherche No $1 \mathrm{~A}$ sur la prévention des maladies cardio-vasculaires, sont maintenant aussi disponibles en traduction française.

Le manuel «Enseigner la santé» est destiné à fournir aux praticiens de l'information et de l'éducation en matière de santé une vue d'ensemble succincte des principes, des possibilités et de la planification d'une campagne de prevention. Il donne aussi des moyens pratiques pour mettre en cuvre ces principes en proposant des méthodes de travail et des exemples de documents éducatifs.

Le PNR 1A a comporté deux expérimentations de programmes d'éducation à la santé, à Aarau et à Nyon. Ces deux actions, pareilles par leurs buts et par l'ampleur des moyens mis en ceuvre, ont été menées de façon relativement différente, selon les orientations choisies par les équipes locales. L'une a plutôt mis l'accent sur l'utilisation des mass media et la réalisation de documents éducatifs (Aarau), l'autre s'est davantage tournée vers une forme de pédagogie pratiquée en petits groupes (Nyon). Les deux démarches ont réussi à influencer positivement les comportements liés à la santé et à entraîner une diminution des risques dans les collectifs touchés.

Les lecteurs francophones peuvent maintenant prendre connaissance des moyens avec lesquel le PNR 1 A a travaillé à Aarau, en se procurant le manuel «Enseigner la santé». Celui-ci peut-être obtenu gratuitement à l'Institut universitaire de médecine sociale et préventive, Bugnon 17, 1011 Lausanne, ou auprès de la maison Sandoz, division publicité, 4002 Bâle, qui a offert cette publication.

Les résultats de l'expérience nyonnaise du PNR 1A, pour leur part, ont fait l'objet d'une présentation détaillée dans le No $1 / 83$ des Cahiers médico-sociaux sous le titre "Prévention des maladies cardio-vasculaires: méthodes d'éducation pour la santé et évaluation - Nyon». Ce document peut également être obtenu à l'Institut universitaire de médecine sociale et préventive de Lausanne.

A partir de 1981, dans les deux villes choisies par le PNR 1A, les programmes de prévention ont continué à se développer et à répondre aux besoins de la collectivité dans le domaine de l'éducation à la santé. Des informations sur ces actions peuvent être obtenues auprès d'«Aarau eusi gsund Stadt», Postfach 486, 5001 Aarau et des Ligues de la santé, avenue de Provence 12, 1007 Lausanne.

\section{F. Gutzwiller \\ Ph. Lehmann \\ IUMSP Lausanne, mai 1984}

\section{Preis des ACS}

für Arbeiten und Veröffentlichungen aus dem Gebiet der Strassenverkehrs-Unfallmedizin

Zur Förderung der Forschungsarbeiten und Studien, die sich mit den medizinischen Problemen des Strassenverkehrs befassen, namentlich
- Verhütung von Gesundheitsschäden, die durch den Verkehr verursacht werden,

- Organisation der ärztlichen Hilfe,

- Erkennen und Behandlung von Verletzungen bei Verkehrsunfällen,

schreibt der Automobil-Club der Schweiz (ACS) Preise aus, dessen Träger in jedem geraden Jahr bestimmt werden.

Die Preissumme beträgt Fr. 7500.-; sie wird in der Regel auf einen 1. Preis von Fr. 5000.- und einen 2. Preis von Fr. 2500.verteilt.

Die Preisträger werden durch einen Ausschuss der Ärztekommission des ACS ausgesucht und der Ärztekommission zur definitiven Stellungnahme vorgeschlagen. Die Ausrichtung des Preises erfolgt durch das Direktionskomitee des ACS.

\section{Bedingungen}

1. Die Arbeiten sollen von Schweizern oder von in der Schweiz tätigen Autoren verfasst sein. Es ist nicht notwendig, dass es sich um Ärzte handelt. Es kann auch die Veröffentlichung einer Arbeitsgruppe berücksichtigt werden.

2. Bereits veröffentlichte Arbeiten können in Betracht gezogen werden. Die Veröffentlichung darf aber nicht mehr als zwei Jahre zurückliegen (für 1984: nicht vor dem 31. Oktober 1982).

3. Die Arbeiten können entweder

- frei yon der Ärztekommission aus dem ihr bekannten Schrifttum bestimmt werden, ohne dass deren Autoren sich um den Preis bewerben;

- von den Autoren an die Ärztekommission des ACS als Bewerbung um den ACS-Preis vor oder nach Veröffentlichung zur Beurteilung eingesandt werden.

4. Es dürfen auch Dissertationen und Habilitationsarbeiten eingeschickt werden. Eine Arbeit darf nicht zweimal eingereicht werden.

5. Die Ärztekommission des ACS behält sich das Recht vor, spezielle Preisfragen für eine bestimmte Periode aufzustellen.

6. Die Ausrichtung des Preises ist an die Bedingung geknüpft, dass die Arbeit in einer von der Ärztekommission des ACS zu bestimmenden Zeitschrift oder Abhandlung veröffentlicht werden kann. Die Namen der Preisträger sowie eine Zusammenfassung der Arbeiten werden sowohl in den Organen des ACS wie auch in der «Schweizerischen Ärztezeitung» veröffentlicht.

7. Es steht der Ärztekommission des ACS frei, in bezug auf die Verteilung der Preise dem Direktionskomitee eigene, von der Regel abweichende Vorschläge zu unterbreiten oder den Verzicht auf die Ausrichtung von Preisen zu beantragen.

8. Die Arbeiten sind in drei Exemplaren bis spätestens 31. Dezember 1984 einzureichen an:

Automobil-Club der Schweiz

Zentralverwaltung

Wasserwerkgasse 39

3000 Bern 13 